\title{
Análisis de la dureza superficial del cuerno en el Toro de Lidia e influencia del enfundado
}

\author{
Analysis of the surface hardness of the Lidia Bull horn and the influence of the horn sheath
}

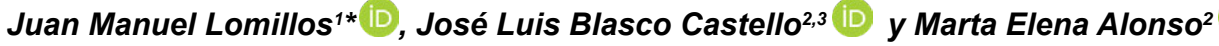 \\ ${ }^{1}$ Departamento de Producción y Sanidad Animal, Salud Pública Veterinaria y Ciencia y Tecnología de los Alimentos, Facultad de \\ Veterinaria, Universidad Cardenal Herrera-CEU. Alfara del Patriarca, Valencia, España. ${ }^{2}$ Departamento de Producción Animal, \\ Facultad de Veterinaria de León, Universidad de León. Campus de Vegazana, León, España. ${ }^{3}$ Veterinario Técnico de vacuno de cebo \\ y pequeños rumiantes de MSD Animal Health - Veterinario de la plaza de toros de Zaragoza. Zaragoza, España.
}

Correo electrónico: juan.lomillos@uchceu.es

\begin{abstract}
RESUMEN
La cornamenta en el toro de lidia (TdL) es uno de los elementos anatómicos más importante del animal, ya que le confiere su carácter ofensivo y a la vez le dota de pureza e integridad. En la actualidad se protege el Cuerno (C) durante el último año de cría del toro con una funda de fibra de vidrio. Con el presente trabajo se pretendió estudiar la dureza del C del TdL analizando la posible influencia del enfundado en la misma. Para ello se han recogido los $C$ de 390 toros (4-5 años -a-) y novillos (3 a) en plazas de toros de España y Francia, contando con un grupo control de individuos no enfundados de 60 animales. Se ha analizado la dureza de las 3 partes del C: cepa, pala y pitón, mediante la utilización de un durómetro PCE-D shore ${ }^{\circledR}$. A su vez, se recogió información biométrica del $\mathrm{C}$, se anotó la presencia de lesiones en la cornamenta y la capa del animal. Se observa que los $C$ de los animales enfundados presentaron una menor dureza superficial, tanto a nivel de la cepa como del pitón. Los $\mathrm{C}$ que mostraron alguna alteración como escobillado o astillado entre otros, la dureza superficial del pitón fue menor. $\mathrm{Y}$, por último, los $\mathrm{C}$ de los toros jaboneros resultaron ser más blandos, fundamentalmente a nivel del pitón, que los pertenecientes a animales con otras capas.
\end{abstract}

Palabras clave: Toro de lidia; raza de lidia; cuernos

\begin{abstract}
The bull's Horn $(\mathrm{H})$ is one elements of the anatomicals part of the animal, since it confers its offensive character and at the same time gives it purity and integrity. Currently the $\mathrm{H}$ is protected during the last year of bull breeding with a fiberglass sheath. With the present work, it has tried to study the hardness of the $\mathrm{H}$ of the fighting bull, analyzing the possible influence of the holster on it. For this, $\mathrm{H}$ of 390 bulls ( $4-5$ years -yr- and steers ( $3 \mathrm{yr}$ ) have been collected in bullfights in Spain and France, with a control group of individuals not covered with 60 animals. The hardness of the three parts of the $\mathrm{H}$, strain, shovel and python has been analyzed, using a PCE-D shore ${ }^{\circledR}$ hardness tester. In turn, biometric information was collected from the $\mathrm{H}$, the presence of lesions on the antlers and the animal's coat was noted. It was observed that the $\mathrm{H}$ of the sheathed animals showed a lower surface hardness both at the level of the strain and the $\mathrm{H}$ tip. The $\mathrm{H}$ that showed some alteration such as brushing or chipp/ing among others defects. The surface hardness of the $\mathrm{H}$ tip was lower. And finally, the $\mathrm{H}$ of soapy bulls turned out to be softer, mainly at the level of the python, than those belonging to animals with other layers.
\end{abstract}

Key words: Lidia bull; lidia cattle; horns 


\section{INTRODUCCIÓN}

El cuerno (C) del toro (Bos taurus) es, sin lugar a duda, un elemento trascendental en la fiesta taurina y en el rito del espectáculo son el icono de integridad y pureza del espectáculo mismo. Sobre esta integridad se ha debatido y debate desde los inicios del toreo. La aproximación veterinaria hacia este aspecto debe realizarse desde un punto de vista técnico y profesional y por tanto, como una estructura anatómica del animal susceptible de sufrir diversas lesiones y patologías [34]. Por ello, es fundamental el conocimiento de sus características anatómicas, histológicas, fisiológicas y patológicas que permitan diagnosticar y explicar las diferentes enfermedades o fraudes que le afecten [1].

La literatura existente sobre los $C$ del TdL no es muy abundante. Los libros clásicos de exterior [2, 32, 33] tratan el tema de las encornaduras como una región anatómica más, pero no se refieren de forma específica al TdL. Otros autores $[6,12]$ se refieren concretamente a dicha raza, pero hacen, más bien, una clasificación según su conformación.

A partir del descubrimiento del fraude del "afeitado", la literatura sobre conformación de los $\mathrm{C}$ y su relación con la capacidad combativa de las reses ha sido más abundante [3, 9, 13, 16, 17, 22-24, 34].

EI C es una producción epidérmica que se sitúa a ambos lados de la región frontal (del testuz), sustentado en el saliente óseo del hueso frontal, adoptando forma cónica y alargada. Se divide en 3 partes: parte proximal o "cepa" (también llamada mazorca), parte media o "pala" y parte distal o "pitón" [14]. Como tal producto óseo, aparecen en su composición minerales como el calcio, hierro, magnesio, sodio, potasio y fosforo, dependiendo su dureza, en gran medida, de la misma [10].

El TdL es un rumiante cavicorne, con $C$ huecos y persistentes y su estructura desde la profundidad a la superficie es la siguiente:

- Proceso cornual (Processus cornuali) saliente óseo del hueso frontal, "clavija ósea" en el argot taurino, constituido por tejido óseo esponjoso envuelto por periostio de tejido óseo compacto. Dicho soporte óseo en su base está limitado por un profundo surco (ranura) vascular. Su superficie, muy rugosa, está llena de pequeños orificios y excavada por numerosos surcos. Dicha excavación corresponde al seno del hueso o divertículo corneal y está en comunicación con la porción caudal del seno frontal (contiene vasos, nervios y tejido conjuntivo en las cavidades grandes).

El epitelio corneal, FIG. 1, de la profundidad a la superficie está conformado por:

- Dermis, corion o capa intermedia de tejido conjuntivo, vascular, nervioso y papilar que recubre la estructura de hueso "clavija ósea" y se adhiere a ella fuertemente por su parte superficial (externa). Nutre a la siguiente capa, tiene aspecto aterciopelado, es roja, delgada y tiene un espesor de aproximadamente 2,5 milímetros ( $\mathrm{mm}$ ). Se denomina comúnmente "membrana queratógena".

- $\quad$ Epidermis, compuesta por tres estratos: basal, espinoso y córneo. Es similar a la de otras zonas cutáneas, pero sin estrato lúcido y con gran desarrollo del estrato córneo, llamado "estuche córneo". Las paredes del "estuche córneo" son delgadas y cortadas en bisel en su base, pero aumentan de grosor hasta terminar en un cono macizo en la punta

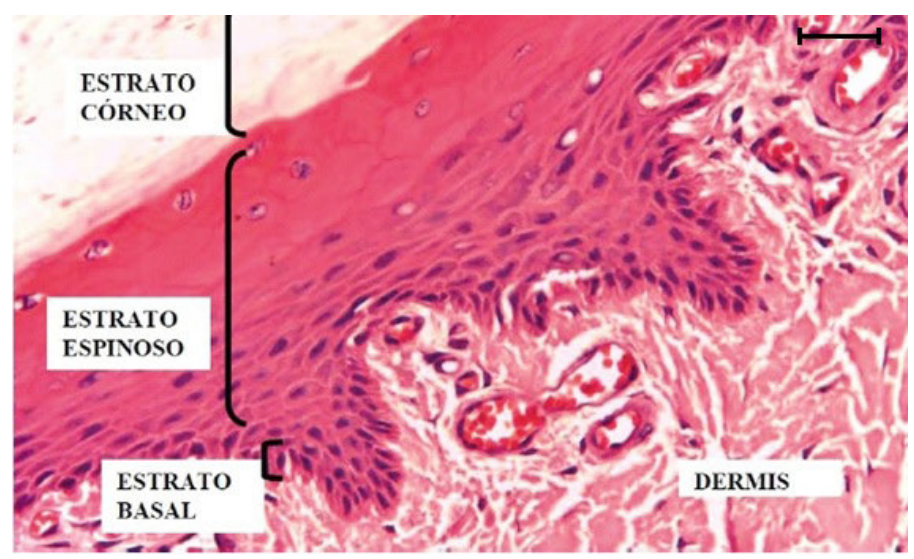

FIGURA 1. Capas del epitelio corneal [1]. Tinción hematoxilinaeosina, 40 aumentos (40x). scale bar (arriba a la derecha): 100 micrómetros. Fuente: Alonso, M.E., Lomillos, J.M., González J.R. (2016).

de tamaño y grosor variable. Este estrato está formado por células completamente queratinizadas (corneocitos), aplanadas, sin núcleos, que sólo conservan restos de orgánulos, ya que prácticamente todo el citoplasma está ocupado por densos paquetes de filamentos de queratina. Estas células se engarzan formando escamas y se organizan a lo largo del estrato córneo de forma concéntrica, debido a la papilaridad de la dermis y las consiguientes interdigitaciones de la dermis. Con el progresivo crecimiento del estrato córneo, la papilaridad de la dermis determina la formación de multitud de cilindros de células queratinizadas que se van disponiendo inclinados en la dirección del eje de crecimiento longitudinal del $\mathrm{C}$ y acaban siendo paralelos a la superficie del mismo, lo que fundamentalmente se hace evidente en la zona del pitón.

Si se observa un esquema del corte mediano del cuerno (FIG. 2) es posible apreciar las dimensiones relativas del hueso o proceso cornual, que termina en la punta del cuerno o pitón formando una línea blanca en el centro del estuche córneo hasta el ápex cornual o punta del pitón.

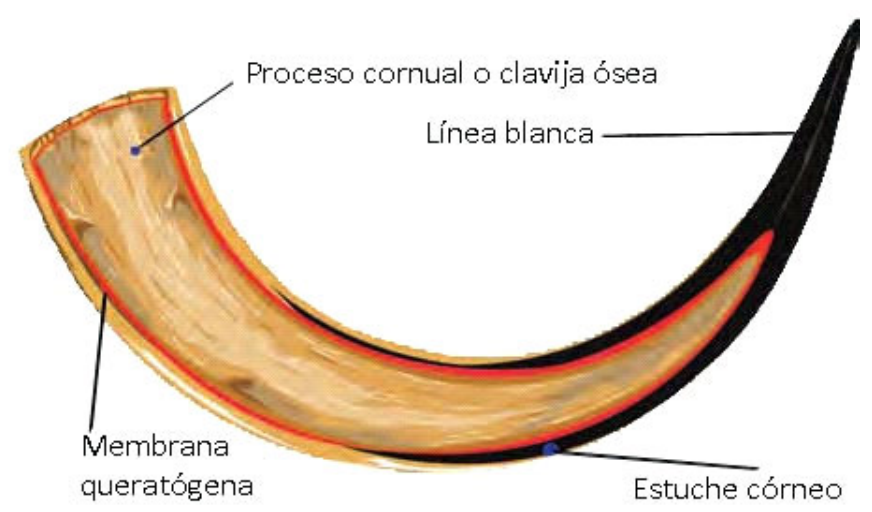

FIGURA 2. Anatomía del cuerno del toro. Corte mediano del cuerno (Fuente: Calvo, 2005) [11] 
Por su parte el esquema superficial (FIG. 3) permite visualizar los surcos circulares o anillos cornuales, que coinciden con cada brote anual de crecimiento córneo. Estos surcos permiten determinar la edad aproximada del animal [24], teniendo en cuenta, en animales maduros, que el primer surco o anillo cornual se cuenta como tres años (a), ya que el primero y el segundo se desdibujan con el tiempo [11].

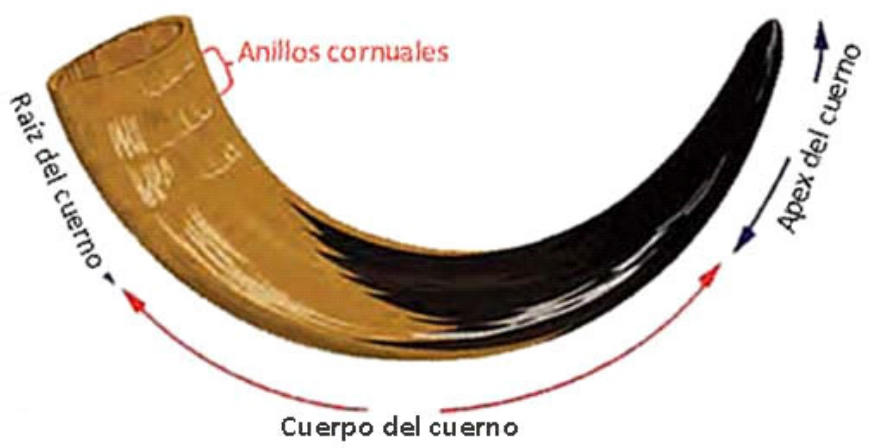

FIGURA 3. Anatomía del cuerno del toro. Vista superficial del cuerno. (Fuente: Calvo, 2005) [11]

La encornadura del toro sufre un riesgo de deterioro, fundamentalmente en el último a de vida, a consecuencia de potenciales peleas, roces, contactos o golpes con el suelo, con árboles, el vallado, comederos o las paredes de las mangas o corrales de manejo [3]. Por ello, en la década de los 60 se popularizó, entre las ganaderías andaluzas, el uso de planchas finas de plomo para el recubrimiento o protección de los $\mathrm{C}$. Estas fundas consistían en láminas muy finas que se moldeaban alrededor del pitón. El método ofrecía gran protección frente al rascado contra el suelo o las encinas, pero el metal lesionaba las puntas de los pitones cuando los toros peleaban.

Posteriormente, en la década de los 80, se cambió el plomo por aluminio y las láminas por un recubrimiento que se adhería al C con pegamento. Estas variaciones no producían lesiones pero el artilugio se desprendía fácilmente [21].

En los años '90 se opta por sustituir el aluminio por Policloruro de Vinilo (PVC), mucho más ligero y manejable, pero este material causaba maceraciones y, en algunos casos, putrefacciones en el tejido córneo [18].

Actualmente se usa un vendaje de fibra de vidrio, fácil de manejar, poroso y que se endurece rápidamente por polimerización con el agua, proporcionando buena consistencia (FIG 4). La técnica consiste en acceder al animal mediante el cajón de inmovilización y envolver el $\mathrm{C}$ con este vendaje para protegerlo de cualquier agresión o roce. La parte distal del C, es decir el pitón, se refuerza en muchos casos con un material más duro, tubos metálicos o similares, con el fin de disminuir el desgaste de la zona apical [27, 28].

La encornadura queda aumentada de grosor por la funda y el pitón romo, lo cual disminuye el efecto de las cornadas entre animales en un $90 \%$ y, además, mejora su manejo para vacunaciones, desparasitaciones y otros tratamientos, puesto que se reducen los riesgos de deterioro de las defensas al pasar los animales por las mangas de manejo [20].

Existe un debate sobre el uso legítimo de las fundas ante la evidente manipulación de los $\mathrm{C}$ en los momentos de la colocación y retirada del vendaje. Sus defensores argumentan que esta práctica tiene el objetivo de conservar la integridad de los pitones, mientras que la legislación vigente (Reglamento de Espectáculos Taurinos) habla de que el ganadero debe asegurar la "intangibilidad de las defensas del toro".

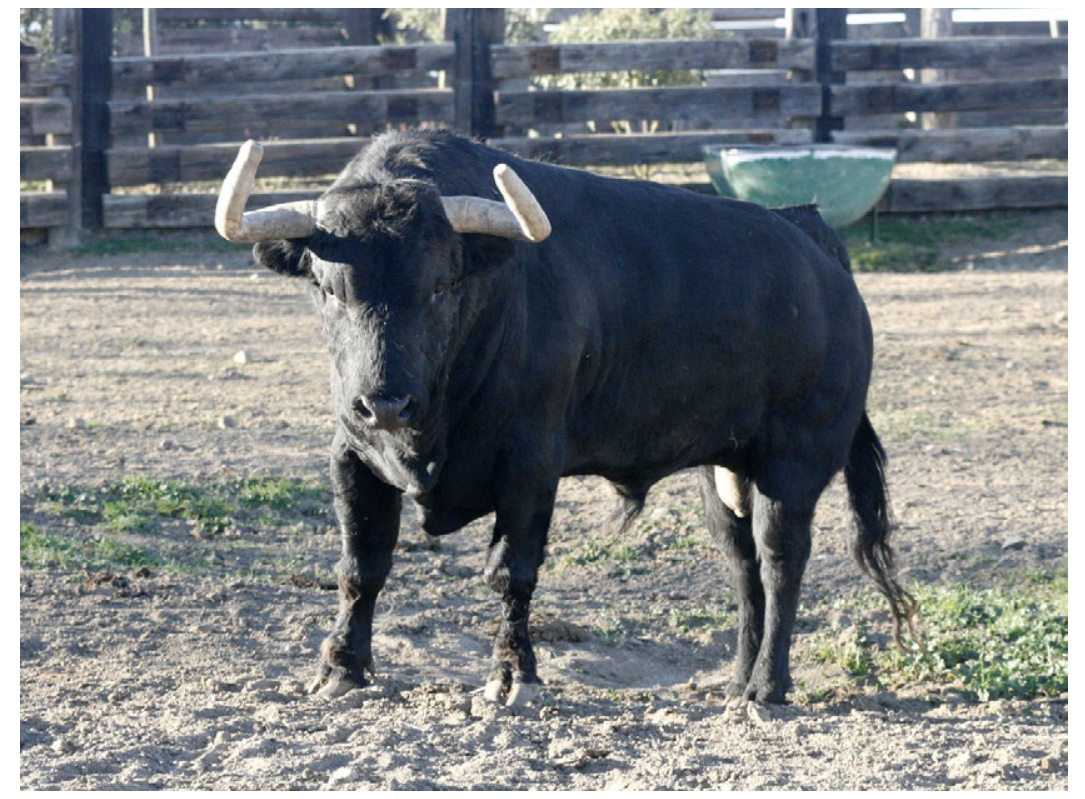

FIGURA 3. Macho de lidia con los cuernos enfundados.

(Fotografía: Julio Cesar Sánchez, 2018). 
Igualmente se desconoce cómo afecta este tipo de manipulación del $\mathrm{C}$ a su estructura y por ende a su dureza. Algunos autores han observado una necrosis de la dermis cornual, con pérdida de la estructura ósea y aparición de espacios huecos en el interior del C, que podrían reducir su resistencia [18].

En cuanto a la dureza superficial del C, se han llevado a cabo algunos pequeños estudios de aproximación al efecto del enfundado que reflejan conclusiones dispares [19, 28, 29, 31, 32]. Por todo ello, el objetivo de este trabajo fue arrojar luz sobre el efecto del enfundado, sobre la dureza del $\mathrm{C}$, con una amplia muestra de animales y con la metodología de análisis adecuada para establecer conclusiones sólidas.

\section{MATERIALES Y MÉTODOS}

Se han analizado los dos $C$ de 390 toros de 4-5 a y novillos de 3 a, lidiados en plazas de toros de España y Francia, contando con un grupo control de individuos no enfundados (NE) de 60 animales.

Los animales enfundados fueron divididos en dos grupos en función del tiempo en el que tuvieron puestas las fundas: $\mathrm{E} 1=6-9$ meses (mes), $\mathrm{E} 2=9-12$ mes

Se ha analizado la dureza superficial de las tres partes del C: cepa, pala y pitón, mediante la utilización de un durómetro modelo PCE-D shore® (PCE Ibérica S.L., España).

A su vez, se recogió información biométrica del C, se anotó la presencia de lesiones en la cornamenta y la capa del animal.

Una vez que los animales fueron lidiados se accedió al desolladero donde se tomaron "in situ" los siguientes datos:

- variables biométricas: longitud externa, interna del C y la distancia entre $\mathrm{C}$, medidas con cinta métrica (marca NEOFERR $®$, modelo fibra 5 metros España), diámetros vertical y horizontal en la cepa, pala y pitón mediante calibre.

- dureza de las 3 partes del cuerno, cepa (inicio), pala (parte media) y pitón (punta o parte final), mediante la utilización del durómetro PCE-D shore ${ }^{\circledR}$ (FIG. 5). Inicialmente se iba a utilizar un modelo digital, sin embargo, tras consultar con los ingenieros de la casa comercial, se optó por utilizar el modelo analógico por su mayor fiabilidad y repetibilidad en la utilización.

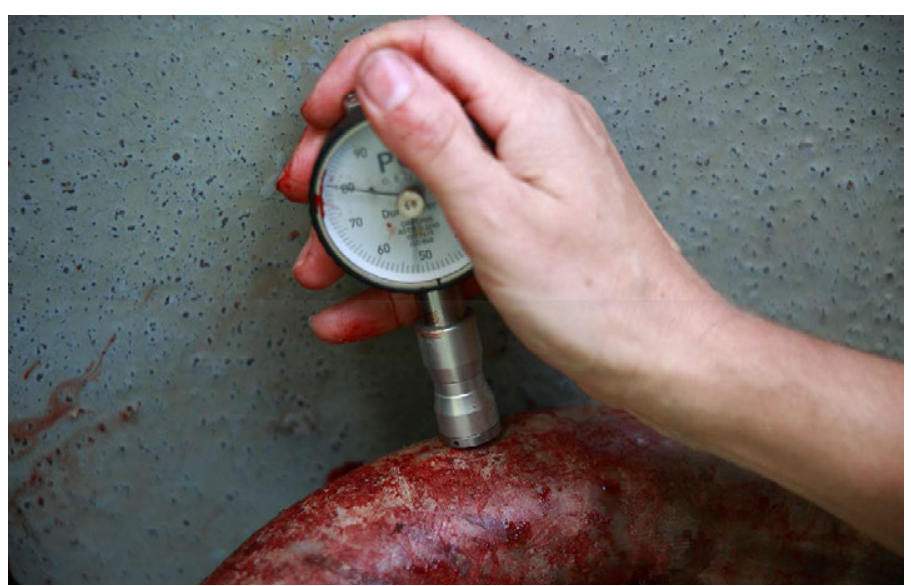

FIGURA 5: Midiendo la dureza superficial en la pala

\section{Análisis estadístico}

Los datos recogidos fueron sometidos a pruebas estadísticas no paramétricas analizando las diferencias entre grupos con las pruebas Newman-Keuls y Kolmogorov-Smirnov, escogiéndose ésta última para presentar los datos por proporcionar los valores medios de cada variable, lo cual facilita la labor de interpretación de los resultados. Todos los cálculos estadísticos se han hecho con el programa IBM $®$ SPSS $®$ statistic, Ver. 19.0 package for Windows.

\section{RESULTADOS Y DISCUSIÓN}

En la TABLA I se exponen las medias de dureza obtenidas para cada variable estudiada en unidades Shore D. Se presentan los resultados en primer lugar del $\mathrm{C}$ derecho y después del C izquierdo.

Se aprecia cómo los $\mathrm{C}$ de los toros que no habían estado enfundados presentaron mayor dureza, tanto a nivel de la cepa como del pitón en ambos C, apoyando la tesis de estudios anteriores [31].

Se realizó una matriz de correlación lineal entre los valores de dureza superficial y los biométricos, en un intento de comprobar qué valores biométricos presentan influencia sobre los de dureza (TABLA II). A nivel de la cepa influye positivamente la longitud medida por la curvatura mayor (longitud externa), el diámetro vertical de la

TABLA I

Kolmogorov - Smirnov. Test de dureza superficial

\begin{tabular}{cccccccc}
\hline & $\begin{array}{c}\text { Grupo } \\
\text { Enfundados } \\
(\mathbf{n})\end{array}$ & $\begin{array}{c}\text { Grupo no } \\
\text { enfundados } \\
(\mathbf{n})\end{array}$ & $\begin{array}{c}\text { Media } \\
\text { Enfundados } \\
\text { (Shore D) }\end{array}$ & $\begin{array}{c}\text { Media } \\
\text { No Enfundados } \\
\text { (Shore D) }\end{array}$ & $\begin{array}{c}\text { Desviación } \\
\text { Estandar } \\
\text { Enfundados. }\end{array}$ & $\begin{array}{c}\text { Desviación } \\
\text { Estandar } \\
\text { No Enfundados }\end{array}$ & P valor \\
\hline Dureza Cepa & 272 & 118 & 77,650 & 81,650 & 9,37 & 8,00 & $\mathrm{P}<0,005$ \\
Dureza cepa derecha & 272 & 118 & 80,840 & 83,480 & 8,97 & 7,13 & $\mathrm{P}>0,100$ \\
Dureza pala derecha & 268 & 116 & 81,540 & 83,690 & 10,30 & 10,31 & $\mathrm{P}<0,025$ \\
Dureza pitón derecho & 253 & 110 & 77,480 & 81,190 & 9,23 & 8,43 & $\mathrm{P}<0,010$ \\
Dureza pala izquierda & 255 & 110 & 80,800 & 80,640 & 8,69 & 8,74 & $\mathrm{P}>0,100$ \\
Dureza pitón izquierdo & 244 & 110 & 81,740 & 84,990 & 10,33 & 8,59 & $\mathrm{P}<0,025$ \\
\hline
\end{tabular}




\section{Matriz de correlación lineal entre dureza superficial y parámetros biométricos del cuerno}

\begin{tabular}{cccc|cccc}
\hline Medida & \multicolumn{3}{c}{ Dureza } & Medida & \multicolumn{3}{c}{ Dureza } \\
& Cepa & Pala & Pitón & Cepa & Pala & Pitón \\
\hline Long Externa & 0,216 & 0,261 & 0,213 & Diametro Horizontal Pitón & $-0,089$ & $-0,085$ & $-0,096$ \\
Long Interna & 0,080 & 0,112 & 0,141 & Dureza Cepa & 1,000 & 0,708 & 0,465 \\
Diametro Vertical Cepa & 0,161 & 0,184 & 0,139 & Dureza Pala & 0,708 & 1,000 & 0,448 \\
Diametro Horizontal Cepa & $-0,094$ & $-0,073$ & $-0,104$ & Dureza Pitón & 0,465 & 0,448 & 1,000 \\
Diametro Vertical Pala & $-0,054$ & $-0,045$ & $-0,184$ & Distancia entre Cuernos & $-0,024$ & $-0,001$ & $-0,046$ \\
Diametro Horizontal Pala & $-0,069$ & $-0,071$ & $-0,042$ & Peso Cuerno Derecho & 0,378 & 0,343 & 0,231 \\
Diametro Vertical Pitón & $-0,083$ & $-0,036$ & $-0,078$ & Peso Cuerno Izquierdo & 0,390 & 0,350 & 0,267 \\
\hline
\end{tabular}

misma y el peso de ambos C. La misma tendencia se observa en la dureza de la pala y el pitón incluyendo la medida tomada también en la curvatura menor. Así pues, los $\mathrm{C}$ de mayores dimensiones resultan más duros superficialmente. Posiblemente estos $C$ de mayor magnitud pertenezcan a animales de mayor edad, pero esta variable no la hemos tenido en cuenta en este estudio $[8,15]$.

Igualmente se realizó un análisis de Kolmogorov-Smirnov para comprobar la influencia del tiempo que los animales llevaron puestas las fundas protectoras del $\mathrm{C}$, considerando dos grupos: el primero para los que las llevaron entre 6 y 8 mes y el segundo para los de 9 a 12 mes o más. Los resultados (TABLA III) sólo presentaron diferencias significativas para el valor de la dureza superficial del pitón derecho, que fue superior en los animales que llevaban más tiempo enfundados (grupo E2), por lo que no se puede concluir que una mayor permanencia de las fundas produzca menor dureza superficial.

Se realizó un estudio descriptivo de la incidencia de alteraciones en los pitones teniendo en cuenta las encontradas por otros autores $[4,5,7,28,30]$, cuyos resultados se muestran en la TABLA IV. Se comprueba que la incidencia de pitones rotos es mayor en el grupo enfundado, mientras que los escobillados y con pérdida de capas de tejido córneo (pérdida de sustancia) son más frecuentes en el grupo no enfundado.

Seguidamente se estudió la influencia de aparición de alteraciones (astillados, escobillado...) en la punta del pitón (Grupo 0) sobre los valores de dureza superficial de $\mathrm{C}$ derecho (TABLA V) e izquierdo (TABLA VI). Se comprobó que los animales con dichas alteraciones presentaban una dureza menor que los que no presentaban alteraciones (Grupo 1), pero sólo a nivel del pitón, lo cual parece indicar que la causa de la alteración no sea una menor dureza en toda la estructura del C $[18,26]$.

Por último, se realizaron ANOVA Kruskal-Wallis para detectar diferencias en función de la coloración del pelaje de los animales sobre los valores de dureza superficial que se muestran en las FIGS. 6,7 y 8 .

TABLA III

Kolmogorov-Smirnov. Test de dureza superficial en función del tiempo de permanencia de las fundas

\begin{tabular}{cccccccc}
\hline & $\begin{array}{c}\text { E1 } \\
(\mathbf{n})\end{array}$ & $\begin{array}{c}\text { E2 } \\
(\mathbf{n})\end{array}$ & $\begin{array}{r}\text { Media E1 } \\
\text { (Shore D) }\end{array}$ & $\begin{array}{r}\text { Media E2 } \\
\text { (Shore D) }\end{array}$ & $\begin{array}{r}\text { Desviación } \\
\text { Estandar E1 }\end{array}$ & $\begin{array}{c}\text { Desviación } \\
\text { Estandar E2 }\end{array}$ & P valor \\
\hline Dureza Cepa & 74 & 94 & 80,649 & 81,287 & 7,40 & 5,03 & $\mathrm{P}>0,10$ \\
Dureza Pala & 74 & 94 & 83,635 & 84,319 & 7,92 & 6,11 & $\mathrm{P}>0,10$ \\
Dureza Pitón & 74 & 94 & 82,527 & 84,734 & 9,99 & 10,40 & $\mathrm{P}<0,05$ \\
Dureza Cepa & 69 & 85 & 80,812 & 80,624 & 6,77 & 7,28 & $\mathrm{P}>0,10$ \\
Dureza Pala & 69 & 85 & 84,913 & 83,471 & 7,05 & 6,61 & $\mathrm{P}>0,10$ \\
Dureza Pitón & 69 & 84 & 81,391 & 84,762 & 12,40 & 10,25 & $\mathrm{P}>0,10$ \\
\hline
\end{tabular}

Grupos en función de la duración del enfundado: Grupo E1 = 6 - 9 meses; E2 = 9 - 12 meses. 
TABLA IV

Animales con alteraciones en el cuerno

\begin{tabular}{cccccc}
\hline & \multicolumn{4}{c}{ Lesión encontrada } \\
\cline { 2 - 5 } & Escobillado (\%) & Astillado (\%) & Pitón roto (\%) & Cuerno desgastado (\%) & Pérdida sustancia (\%) \\
\hline Enfundados $(\mathrm{n}=233)$ & 0,43 & 10,30 & 6,00 & 7,30 & 1,72 \\
No enfundados $(\mathrm{n}=100)$ & 5,00 & 13,00 & 2,00 & 9,00 & 4,00 \\
\hline
\end{tabular}

TABLA V

Kolmogorov - Smirnov. Test de dureza superficial en función de la presencia o ausencia de alteraciones. Pitón derecho

\begin{tabular}{ccccccc}
\hline & Media & Media & Desviación \\
& grupo 1 & grupo 0 & $\begin{array}{c}\text { Estandar } \\
\text { grupo 1 }\end{array}$ & $\begin{array}{c}\text { Desviación } \\
\text { Estandar } \\
\text { grupo 0 }\end{array}$ & $\begin{array}{c}\text { Enfundado } \\
\text { grupo 1 } \\
\mathrm{n}\end{array}$ & $\begin{array}{c}\text { No Enfundado } \\
\text { grupo 0 } \\
\mathrm{n}\end{array}$ \\
\hline Dureza Cepa & 79,706 & 80,188 & 8,94 & 7,62 & 250 & 85 \\
Dureza Pala & 82,452 & 84,294 & 8,11 & 6,57 & 250 & 85 \\
Dureza Pitón & 86,308 & 72,934 & 8,08 & 10,36 & 250 & $\mathrm{P}>0,100$ \\
\hline
\end{tabular}

TABLA VI

Kolmogorov - Smirnov. Test de dureza superficial en función de la presencia o ausencia de alteraciones. Pitón izquierdo

\begin{tabular}{|c|c|c|c|c|c|c|c|}
\hline & $\begin{array}{c}\text { Media } \\
\text { grupo } 1\end{array}$ & $\begin{array}{c}\text { Media } \\
\text { grupo } 0\end{array}$ & $\begin{array}{c}\text { Desviación } \\
\text { Estandar } \\
\text { grupo } 1\end{array}$ & $\begin{array}{c}\text { Desviación } \\
\text { Estandar } \\
\text { grupo } 0\end{array}$ & $\begin{array}{c}\text { Enfundado } \\
\text { grupo } 1 \\
\text { n }\end{array}$ & $\begin{array}{c}\text { No Enfundado } \\
\text { grupo } 0 \\
\text { n }\end{array}$ & $P$ \\
\hline Dureza Cepa & 79,521 & 79,645 & 8,87 & 9,12 & 238 & 76 & $P>0,100$ \\
\hline Dureza Pala & 82,895 & 82,105 & 8,05 & 8,41 & 238 & 76 & $P>0,100$ \\
\hline Dureza Pitón & 87,097 & 71,761 & 10,33 & 6,71 & 238 & 67 & $P<0,001$ \\
\hline
\end{tabular}

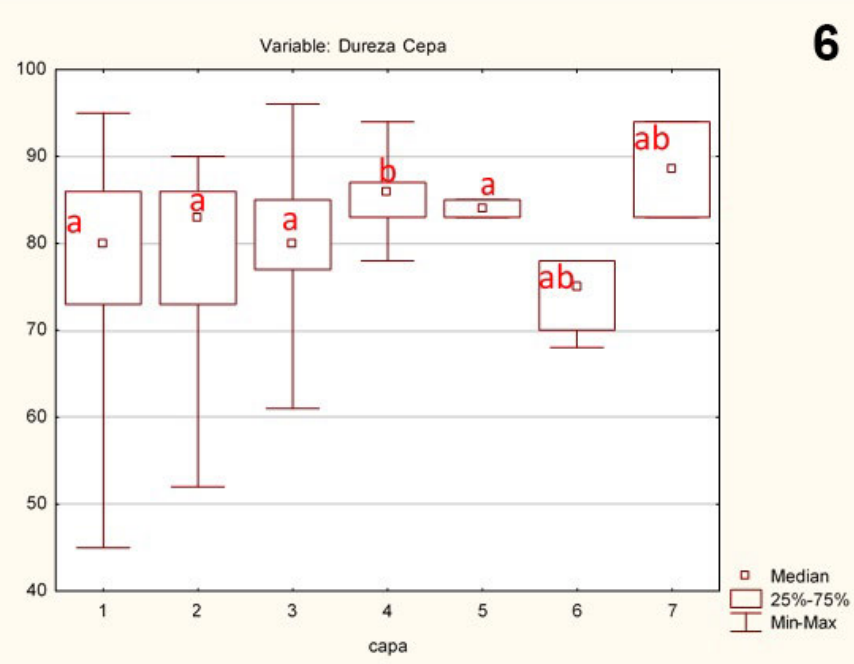

FIGURA 6. Kruskal-Wallis ANOVA (H [6, n = 314] = 13,69589. $P=0,0332$ ) de la dureza de la cepa del cuerno en funcion de la capa. 1 = Negro, 2 = Colorado, 3 = Castaño, 4 = Cárdeno, 5 = Burraco, 6 = Jabonero, 7 = Sardo. Letras distintas indican diferencias significativas a nivel de $P<0,05$

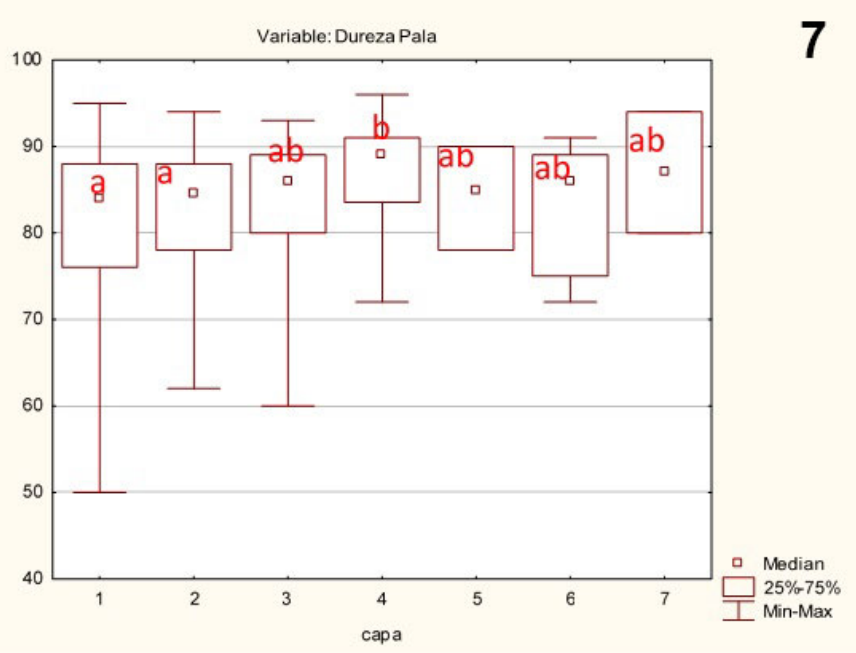

FIGURA 7. Kruskal-Wallis ANOVA (H $[6, n=314]=12,00876$. $P=0,0498$ ) de la dureza de la pala del cuerno en funcion de la capa. 1 = Negro, 2 = Colorado, 3 = Castaño, 4 = Cárdeno, 5 = Burraco, 6 = Jabonero, 7 = Sardo. Letras distintas indican diferencias significativas a nivel de $P<0,05$ 


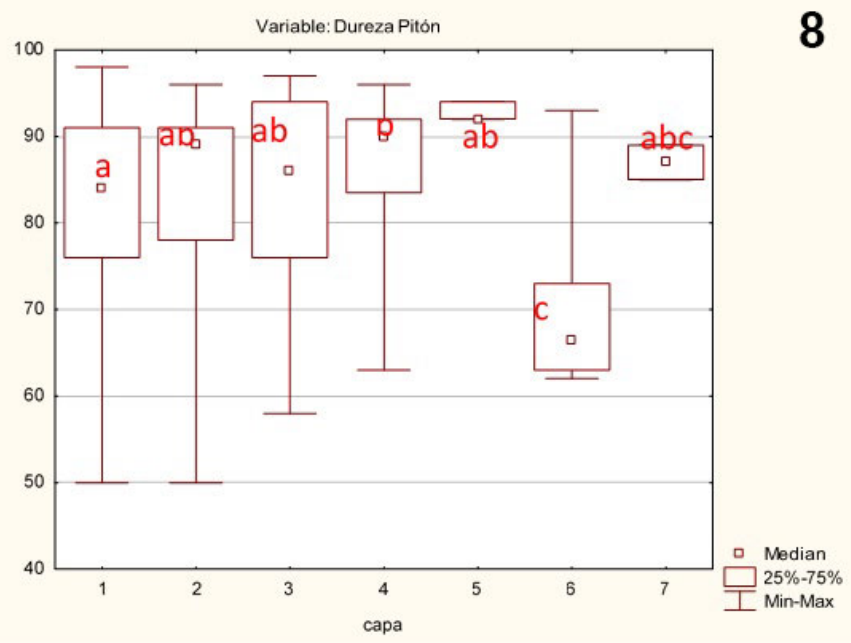

FIGURA 8. Kruskal-Wallis ANOVA (H [6, n = 314] = 17,84923. $P=0,0066$ ) de la dureza del pitón del cuerno en funcion de la capa. 1 = Negro, 2 = Colorado, 3 = Castaño, 4 = Cárdeno, 5 = Burraco, 6 = Jabonero, 7 = Sardo. Letras distintas indican diferencias significativas a nivel de $P<0,05$

Como se ha podido comprobar, los $\mathrm{C}$ de animales cárdenos (Grupo 4) presentaron mayor dureza, mientras en los toros jaboneros (Grupo 6) tuvieron valores inferiores a los de las demás pintas. De los animales sardos (Grupo 7) no se pueden extraer conclusiones, pues la falta de significación puede ser debida al escaso número de animales que integran este grupo. En cualquier caso, cada pelaje está asociado a un determinado encaste y puede que la genética propia de estas líneas genéticas influya en la propia dureza y características propias de sus encornaduras [15, 25, 31].

\section{CONCLUSIONES}

Los $\mathrm{C}$ de los animales enfundados presentaron una menor dureza superficial, tanto a nivel de la cepa como del pitón.

Los $C$ de mayores dimensiones resultan más duros superficialmente, posiblemente como consecuencia de la edad del animal.

Se comprueba que los $C$ enfundados presentan más porcentaje de pitones rotos, mientras que el resto de las alteraciones como escobillados y pérdidas de capas de tejido córneo (pérdida de sustancia) son más frecuentes en el grupo no enfundado.

Los $\mathrm{C}$ de los toros jaboneros resultaron ser más blandos, fundamentalmente a nivel del pitón, que los pertenecientes a animales con otros pelajes.

\section{REFERENCIAS BIBLIOGRÁFICAS}

[1] ALONSO, M.E.; LOMILLOS, J.M.; GONZÁLEZ, J.R. LoS cuernos del toro de lidia. Descripción anatómica. En: La cornamenta del toro de lidia análisis de su integridad y efecto del enfundado. Ed. Eolas Ediciones, León, España. 166 pp. 2016.

[2] APARICIO-SÁNCHEZ, G. Lesiones de la cornamenta del toro. En: Exterior de los grandes animales domésticos. Ed. Imprenta Moderna, Córdoba, España. 324 pp. 1960.
[3] APARICIO, J.B.; PEÑA, F.; BARONA, L.F. El fraude del afeitado. En: Estudio de las encornaduras del Toro de Lidia. Ed. Junta de Andalucía. Córdoba. 154 pp. 2000.

[4] BALLESTEROS-MORENO, E. Examen de cuernos y análisis de afeitado. En: El asta de la res de Lidia, su reconocimiento y fraudes. Ed. Propia. Zaragoza, España. 62 pp. 1983.

[5] BARAONA, L.F.; CUESTA, A.E.; PEÑA, F; APARICIO, J.B. Aplicación del análisis de imagen al estudio exteriorista de las encornaduras del Toro de Lidia. V Simposio Nacional del Toro de Lidia. Zafra, 10/24-26. España. Pp 298-302. 2001.

[6] BARGA, R.; JORDANO, D. Biometría de las astas de toros de lidia y dictamen acroqueratómico (afeitado). Ed. Secretaría General Técnica. Ministerio del Interior. Madrid, España. Pp 45-58. 1997.

[7] BARGA, R. Anatomía del toro de lidia. En: El Toro de Lidia. Ed. Alianza Editorial. Madrid, España. 420 pp. 1995.

[8] BLASCO, J.L.; ESCOBEDO, J.M. Telemetría digital aplicada al estudio del crecimiento del asta en el toro de lidia. II Congreso Mundial de Veterinaria Taurina. Córdoba 05/16-18. España. Pp 255-259. 1997.

[9] BOBED, L. Estudios sobre crecimiento del asta en reses de lidia. Universidad de Zaragoza. Zaragoza, España. Tesis de Grado. Pp 133-167. 1982.

[10] CABANAS, J.M.; GUALDA, M.J.; ROSA, M.; DEL PINO, J. 1994. Estudio de la composición mineral de las astas del Toro de lidia. I Congreso Mundial de Veterinaria Taurina. Zaragoza 05/12-15 España. Pp 65-69. 1994.

[11] CALVO, L.A. Anatomía del cuerno. En: Escuela gráfica de toros. Ed. Colegio Oficial de Veterinarios de Valladolid, Valladolid, España. 272 pp. 2005.

[12] CossíO, J.M. Encornadura del toro de lidia. En: Los toros: tratado técnico e histórico. Ed. Espasa Calpe, Madrid, España. 700 pp. 1967.

[13] EZPELETA, E. Biometría de los cuernos de las reses de lidia. IV Symposium Nacional del Toro de Lidia. Zafra, 10/26-28. España. Pp 225-227. 1999.

[14] FERNÁNDEZ, J. Atlas gráfico de pelajes y encornaduras. Curso básico de Espectáculos Taurinos. Ed. Colegio de Veterinarios de Madrid. Madrid 15-17/05 España. 96 pp. 2009.

[15] FUENTE, D; BALLESTEROS, JP; GUERRA, J; MORALES, J; CARPINTERO, M; DURÁN, M; FERNÁNDEZ, C; FERNÁNDEZ, J; FLORES, B; HEBRERO, C; MORENO, F; URQUÍA, JJ. Estudio estadístico de los cuernos lidiados durante las temporadas 1998 y 1999 en la plaza de toros de las Ventas en función del encaste de procedencia e importancia de la lupa binocular en el análisis de los mismos. IV Simposio Nacional del Toro de Lidia. Zafra, 10/26-28. España. Pp 249-253. 1999.

[16] FUENTE, D; GUERRA, J; HEBRERO, C; MORALES, J. Estudio del crecimiento del asta de las reses lidiadas en la plaza de toros de las ventas durante las temporadas taurinas 1995-1996. III Simposium Nacional del Toro de Lidia. Zafra, 10/24-27. España. Pp 139-144. 1997a. 
[17] FUENTE, D; GUERRA, J; HEBRERO, C; MORALES, J; FERNÁNDEZ, C. Estudio por encastes de las astas de toros lidiados en la plaza de toros de las Ventas de Madrid. II Congreso Mundial de Veterinaria Taurina. Córdoba 05/16-18. España. Pp 163-166. 1997b.

[18] GÓMEZ, A. Patología de las astas. II Congreso Mundial de Veterinaria Taurina. Córdoba 05/16-18. España. Pp 109-110. 1997.

[19] HORCAJADA, FJ; FRENÁNDEZ, C; ORTUÑO, S; PIZARRO, $M$. Diferencias físicas de comportamiento entre pitones enfundados y sin enfundar con vendas de resina de fibra de vidrio: prueba de compresión simple. IX Simposium Nacional del Toro de Lidia. Zafra, 10/25-28. España. Pp 237-242. 2009.

[20] LIRA, F. Avances en el cuidado y protección de las defensas del Toro de lidia. En: Manual de manejo y nutrición del Toro de Lidia. Tomo II. García,JJ; Olmedo,S; Rodríguez,L (Eds). Junta de Castilla y León, Instituto Tecnológico Agrario de Castilla y León (ITACYL). Pp 28-43. 2008.

[21] LOMILLOS, J.M.; ALONSO, M.E.; GAUDIOSO, V. Análisis de la evolución del manejo en las explotaciones de toro de lidia. Desafíos del sector. Rev. ITEA. 109(1): 49-68. 2013.

[22] LLORENTE, J. Aportaciones técnicas a la actuación pericial veterinaria en las corridas de toros. Estudio biométrico del asta y constantes bioquímicas hemáticas. Universidad de Zaragoza. Zaragoza, España. Tesis de Grado.120 pp. 1980.

[23] MARTÍN, R. Estudio anatómico y biométrico de la cornamenta del toro de lidia. Med. Vet. 1: 545-553. 1984.

[24] MAUBON, P. Crecimiento córneo. La corne du taureau de combat. Escuela veterinaria de Alford. Universidad de Alfort, France. Tesis de Grado. 123 pp. 1956.

[25] MENÉNDEZ, R.; DURÁN, J.M.; URQUÍA, J.J. Longitud de los cuernos de toros lidiados en la plaza de las Ventas durante la temporada 2009 y su relación con el encaste al que pertenecen. IX Simposium Nacional del Toro de Lidia. Zafra, 10/25-28. España. Pp 189-193. 2009.
[26] MOZOS, E. Avances en el estudio de los cuernos del toro de Lidia y su patología. IV Congreso Mundial Taurino de Veterinaria. Salamanca. 20-22/09. España. Pp 21-22. 2002.

[27] PIZARRO, M.; CARCELLER, H.; ALONSO, R.; HORCAJADA, J.; HEBRERO, C. Utilización de fundas en cuernos I: Colocación e incidencia en el reconocimiento y comportamiento. VI Congreso Mundial de Veterinaria Taurina. Murcia 11/06-08 España. Pp 175-178. 2008a.

[28] PIZARRO, M.; ALONSO, R.; ORTUÑO, S.; FERNÁNDEZ, C. Utilización de fundas en cuernos II: Posible modificación de la estructura y consistencia. VI Congreso Mundial de Veterinaria Taurina. Murcia 11/06-08 España. Pp 179-182. 2008b.

[29] PIZARRO, M.; HORCAJADA, F.J.; FERNÁNDEZ, C.; ORTUÑO, S. Diferencias estructurales entre pitones enfundados y sin enfundar con vendas de resina de fibra de vidrio. IX Simposium Nacional del Toro de Lidia. Zafra, 10/25-28. España. Pp 285-288. 2009.

[30] SALAMANCA, P. Alteraciones externas encontradas en cuernos de Toros de Lidia que habían sido enfundados previamente a su participación en festejos taurinos. IX Simposium Nacional del Toro de Lidia. Zafra, 10/25-28. España. Pp 183-184. 2009a.

[31] SALAMANCA, P. Análisis de la dureza en cuernos de machos de Lidia de tres y cuatro años de edad comparando el factor encaste. IX Simposium Nacional del Toro de Lidia. Zafra, 10/25-28. España. Pp 263-274. 2009b.

[32] SAÑUDO, C. Morfología de la raza de lidia. En: Valoración morfológica de los animales domésticos. Ed. Ministerio de Medio Ambiente y Medio Rural y Marino. Madrid, España. 859 pp. 2009.

[33] SOTILLO, F.; RAMÍREZ, A.R.; SOTILLO, J.L. Biotipología del Toro de Lidia. En: Producciones equinas y de ganado de Lidia, Cap. XV. Zootecnia, bases de producción animal, Tomo XI. Ed. Mundiprensa. Madrid, España. Pp 235-241. 1996.

[34] TRILLO, F. Estudio métrico del asta del toro de lidia y su aplicación práctica. Arch. Zoot. 39(10): 1. 1961. 